\title{
A THEOREM ON LOCALLY EUCLIDEAN GROUPS
}

\author{
G. D. Mostow ${ }^{1}$
}

1. Let $G$ be a connected locally compact group and let $G^{\prime}$ denote the closure of the commutator subgroup of $G . G^{\prime}$ is called the derived subgroup of $G$. Consider the derived series of $G$, that is, the sequence of subgroups $G_{0}, G_{1}, \cdots, G_{n}, \cdots$ where $G_{0}=G$ and $G_{n+1}=G_{n}^{\prime}$. Each $G_{n}$ is connected and this sequence becomes stationary at some finite stage, ${ }^{2}$ that is, for some $n, G_{n}=G_{n+1}$. We define $G_{n}$ to be the "core" of the group. The purpose of this paper is to prove the following:

TheOREM. Let $G$ be a connected locally euclidean group and let $C$ be its core. Then $G / C$ is a Lie group. ${ }^{3}$

$G / C$ is clearly locally connected and solvable, that is, its core is the identity element. If we knew that $G / C$ were finite-dimensional, then it would follow from a theorem of Iwasawa ${ }^{2}$ that $G / C$ is a Lie group. The difficulty in this approach is the lack of information about the finite dimensionality of $G / C$. The object of this paper is to circumvent this difficulty.

2. Simple connectivity. Let $A, B$ be locally connected topological spaces (that is, each point is contained in arbitrarily small connected neighborhoods) and let $f$ be a continuous mapping of $A$ onto $B$. A neighborhood $U \subset B$ is said to be "covered evenly" by $f$ if $f$ maps each component of $f^{-1}(U)$ homeomorphically onto $U$. The pair $(A, f)$ is called an "even covering" of $B$ if each point of $B$ has a neighborhood which is evenly covered by $f$. We adopt the following definition.

Definition. A connected locally connected space is called simply connected if every even covering of it is univalent. ${ }^{4}$

This definition is equivalent to the usual one (that is, in terms of paths homotopic to a point) for spaces which are arcwise connected, arcwise locally connected, and locally simply connected in the usual sense. The usual definition is inadequate for our purpose, however, since we shall desire the condition of simple connectivity to be preserved under weakly-restricted mappings of a certain type. In fact,

Presented to the Society, October 29, 1949; received by the editors April 9, 1949 and, in revised form, January $6,1950$.

1 O.N.R. Fellowship holder 1948-1949.

${ }^{2} \mathrm{~K}$. Iwasawa, On some types of topological groups, Ann. of Math. (1949).

${ }^{3}$ This theorem asserts as a special case the Chevalley-Iwasawa-Malcev theorem that $a$ solvable locally euclidean group is a Lie group.

4 Cf. C. Chevalley, Lie groups, Princeton University Press, 1946, p. 44. 
our main device is the following lemma.

Lemma 1. Let $A$ be a simply connected space and let $\phi$ be a continuous mapping of $A$ onto a locally connected space $B$. Assume $\phi^{-1}(b)$ is connected for all $b$ in $B$. Then $B$ is simply connected.

Proof. Let $\left(B^{*}, f\right)$ be a covering of $B$. We show that $f$ is univalent.

We first note that if $C$ is a connected subset of $B$, then $\phi^{-1}(C)$ is connected. Next, since $A$ is simply connected, there is a continuous mapping $\phi^{*}$ of $A$ into $B^{*}$ such that $\phi(x)=f\left(\phi^{*}(x)\right)$ for all $x$ in $A .^{5}$ The subset $\phi^{*}(A)$ of $B^{*}$ is open. For suppose $m \in \phi^{*}(A)$. Let $V$ be a neighborhood of $f(m)$ which is evenly covered by $f$. Let $U=f^{-1}(V)$. Then $U$ $=\left\{U_{\alpha}\right\}$, where each of the $U_{\alpha}$ are connected components of $U$ and are neighborhoods in $B$ homeomorphic under $f$ to $V$. Assume that $m \in U_{\alpha_{0}}$. Inasmuch as $\phi^{*}\left(\phi^{-1}(V)\right)$ is connected, included in $U$, and contains a point of $U_{\alpha_{0}}$, it follows that $\phi^{*}\left(\phi^{-1}(V)\right)=U_{\alpha_{0}}$. Hence $\phi^{*}(A)$ is open in $B^{*}$. We show now that $\phi^{*}(A)$ is closed. For if $m$ is in the closure of $\phi^{*}(A)$, any neighborhood of $m$ contains a point of $\phi^{*}(A)$. Let $V^{*}$ be a neighborhood of $m$ such that $V=f\left(V^{*}\right)$ is evenly covered by $f$. Applying the same argument as used above, we see that $\phi^{*}\left(\phi^{-1}(V)\right)$ $=V^{*}$. Hence $m \in \phi^{*}(A)$ and it follows that $\phi^{*}(A)$ is closed. Since $\phi^{*}(A)$ is both open and closed in the connected space $B^{*}, \phi^{*}(A)=B^{*}$. Moreover, for all $b$ in $B, f^{-1}(b)=\phi^{*}\left(\phi^{-1}(b)\right)$ is connected. Since $f^{-1}(b)$ is both discrete and connected, it consists of a single point. Hence $f$ is one-to-one. It follows that $B$ is simply connected.

\section{Proof of the theorem.}

LEMMA 2. Let $G$ be a locally compact group and $V$ a closed normal vector subgroup such that $G / V$ is isomorphic to the additive group of reals. Then there is a one-parameter subgroup $P$ such that $G=P \cdot V$ and the mapping of $G$ onto $P \times V$ given by $p v \rightarrow(p, v)$ is a homeomorphism.

Proof. Let $\Gamma_{x}$ denote the automorphism $v \rightarrow x v x^{-1}$ of $V, x \in G$, $v \in V$. On regarding $V$ as a linear space it is seen that $\left\{\Gamma_{x} \mid x \in G\right\}$ is a connected zero or one-parameter group of linear transformations on $V$. Consequently, there is a linear transformation $A$ of $V$ such that $\Gamma_{x}=e^{A l(x)}$ where $l(x)$ is a continuous homomorphism of $G$ onto the additive group of real numbers. If $x$ is suitably close to the identity $e$ of $G$, the linear transformation $I+e^{A l(x)}$ is nonsingular, $I$ denoting the identity transformation of $V$, and consequently $(x v)^{2}$ $=x^{2}$, that is, $v x v x^{-1}=e$ where $x \in G, v \in V$ implies that $v=e$. It fol-

S See C. Chevalley, loc. cit. p. 50. 
lows that the mapping $x \rightarrow x^{2}$ is one-to-one in some compact neighborhood $U$ of $e$. Let $U^{1 / 2^{n}}$ denote $\left\{x^{1 / 2^{n}} \mid x \in U\right\}$ and let $U_{n}=U \cap U^{1 / 2} \cap \ldots$ $\cap U^{1 / 2^{n}} . u_{n}^{2^{p}} \in U_{n-p}$ if $u_{n} \in U_{n}$ and $n \geqq p$. $U_{n}$ is a compact neighborhood of $e$ and in view of the algebraic structure of $V$ and $G / V$ in compact neighborhoods of their identity elements, $\bigcap_{n=1}^{\infty} U_{n}=\{e\}$. For each $n$, select $x_{n} \in U_{n}-U_{n+1}$. Let $y_{n}=x_{n}^{2^{n}}$. Since $y_{n} \in U-U_{1}$ for all $n$, there is a convergent subsequence $\left\{y^{k^{\prime}}\right\}$ with a limit $\bar{x}$ in the closure of $U-U_{1}$. For any $n$, select now a subsequence $\left\{y_{k^{\prime \prime}}\right\}$ such that $\left\{y_{k^{\prime}}^{1 / \prime^{n}}\right\}$ is convergent. Let $\bar{y}_{n}$ denote $\lim _{k \rightarrow \infty} y_{k^{\prime}}^{1 / \rho^{n}} . \bar{y}_{n} \in U_{n}$. Inasmuch as $y_{k^{\prime \prime}} \rightarrow \bar{y}_{n}^{2^{n}}$ and $y_{k^{\prime}} \rightarrow \bar{x}$, it follows that $\bar{y}^{2}=\bar{x}$. Thus we have found an element $\bar{x} \neq e$ and a compact neighborhood $U$ such that $\bar{x}^{1 / 2^{n}} \in U$ for all $n$.

Let $A$ denote the closure of the subgroup generated by $\left\{\bar{x}_{n}^{1 / 2^{n}} \mid\right.$ all $\left.n\right\}$, and let $E=A \cap U$. Since $E$ is compact, $E V / V$ is a compact neighborhood of zero and thus $A V / V=G / V$. $A$ contains a subgroup $B$ such that $A / B$ is discrete and $B=V_{1}+K_{1}$ where $V_{1}$ is a vector group and $K_{1}$ is compact. $^{6}$ Since $A / B$ is discrete and hence denumerable, $B V / V=G / V$ (by a category argument). On the other hand $K_{1} V / V$ $=\{0\}$ so that $K_{1} \subset V$ and hence $K_{1}=\{e\}$. Thus $B=V_{1}$. Select $P$ to be a one-parameter subgroup of $V_{1}$ which is not in $V_{1} \cap V$. Since

$$
P / P \cap V=P V / V=G / V=\text { Reals, }
$$

$P \cap V=\{e\}$, and thus all elements of $G$ can be expressed uniquely as $p \cdot v, p \in P, v \in V$. Since $V$ is a closed normal subgroup, $\lim _{n \rightarrow \infty} p_{n} v_{n}=\bar{p} \bar{v}$ implies that $p_{n} \rightarrow \bar{p}$ and hence $v_{n} \rightarrow \bar{v}$. Consequently the mapping $p \cdot v \rightarrow(p, v), p \in P, v \in V$, is a homeomorphism of $G$ onto $P \times V$.

Lemma 3. Let $G$ be a connected, locally compact group and $K$ a compact connected normal abelian subgroup, and suppose that $G / K$ is isomorphic to the reals. Then there is a one-parameter subgroup $P$ such that $G=P K$ and the mapping of $G$ onto $P \times V$ given by $p v \rightarrow(p, v)$ is a homeomorphism.

Proof. Observe first that $K$ is central. For the automorphisms of $K$ are in a natural one-to-one correspondence with automorphisms of the character group of $K$, which is discrete. Since the mapping $x \rightarrow \alpha \Gamma_{x}$ of $G$ into char $K$ is continuous, $\Gamma_{x}$ being the conjugation of $K$ by the element $x \in G$ and $\alpha$ being a character of $K, \alpha \Gamma_{x}=\alpha$ for all $x \in G$ and for all characters $\alpha$. It follows immediately that $\Gamma_{x}$ keeps each point of $K$ fixed and thus $K$ is central. Moreover $K$, being connected, is infinitely divisible.

Select now $y \in G-K$. There is obviously an element $y_{1}$ such that $y_{1}^{2} k=y$ for some $k \in K$. Select $l \in K$ such that $l^{2}=k$ and set $x_{1}=y_{1} l$.

\footnotetext{
${ }^{6}$ L. Pontrjagin, Topological groups, Princeton University Press, 1946, p. 161.
} 
Then $x_{1}^{2}=y$. Finally, select a sequence $y_{0}, y_{1}, \cdots, y_{n}, \cdots$ such that $y_{n+1}^{2}=y_{n}, y_{0}=y$ and let $A$ denote the closure of the subgroup generated by $\left\{y_{n} \mid n=1,2, \cdots\right\}$. By the argument employed in the proof of Lemma 2, the desired conclusions are inferred.

On combining Lemmas 2 and 3 one proves without difficulty that if $B$ is a closed, connected, normal, abelian subgroup of $G$ and $G / B$ contains a closed subgroup $R$ isomorphic to the reals, then there is a closed one-parameter subgroup $P$ of $G$ such that $P B / B=R$. (One uses the Pontrjagin decomposition for the connected, locally compact abelian group $B$.) It is clear that $P \cap B$ consists of the identity element alone.

Leмма 4. Let $A, B$ be Lie subgroups of the group $G$ such that for all $g \in G, g=a \cdot b$ uniquely with $a \in A, b \in B$. Assume moreover that $B$ is normal. Then $G$ is a Lie group.

Proof. The mapping $a \cdot b \rightarrow(a, b)$ of $G$ onto $A \times B$ is a homeomorphism since $\lim _{n \rightarrow \infty} a_{n} b_{n}=\bar{a} \bar{b}$ implies that $\lim _{n \rightarrow \infty} a_{n}=\bar{a}, \lim _{n \rightarrow \infty} b_{n}=\bar{b}$. Give $G$ the analytic structure of $A \times B$. Then $G$ is a Lie group, for

$$
\left(a_{1} \cdot b_{1}, a_{2} \cdot b_{2}\right) \rightarrow a_{1} a_{2} \cdot a_{2}^{-1} b_{1} a_{2} b_{2}
$$

is an analytic mapping of $G \times G$ onto $G$. $\left[a^{-1} b a\right.$ is an analytic map of $(a, b)$ since the homomorphism $a \rightarrow \Gamma_{a}: b \rightarrow a^{-1} b a, a \in A, b \in B$ of $A$ into the Lie group of automorphisms of $B$ is analytic by virtue of the theorem that a continuous homomorphism of one Lie group into another is analytic.]

Leмма 5. A simply connected locally compact solvable group is a Lie group.

Proof. We use induction on the length of the series of derived subgroups. Let $G$ be the group and $G=G_{1} \supset G_{2} \supset \cdots \supset G_{n} \supset G_{n+1}=\{e\}$. We may assume $G_{n} \neq\{e\}$. The theorem is true for $n=1$ since then $G$ is abelian and decomposes into the direct product of a vector subgroup $V$ and a compact subgroup $K$. Since $K$ is therefore locally connected, compact, and simply connected, it must consist of the identity element alone so that $G$ is a Lie group.

Assume the theorem to be true for groups whose derived series has length less than $n$. Let $G^{*}=G / G_{n}$ and let $\phi$ denote the natural homomorphism of $G$ onto $G^{*}$. Since $G_{n}$ is connected, $G^{*}$ is simply connected and by the induction assumption $G^{*}$ is a Lie group. Consequently, ${ }^{7}$ there are one-parameter subgroups $P_{1}^{*}, \cdots, P_{s}^{*}$ iso-

7 Cf. C. Chevalley, On the topological structure of solvable groups, Ann. of Math. (1941). 
morphic to the reals and closed analytic subgroups $H_{1}^{*}=G^{*}, H_{2}^{*}, \cdots$, $H_{s+1}^{*}=\left\{e^{*}\right\}, e^{*}$ being the identity element of $G^{*}$, such that $H_{i+1}^{*}$ is normal in $H_{i}^{*}$ and for all $h_{i} \in H_{i}^{*}, h_{i}=u_{i} h_{i+1}$ uniquely with $u_{i} \in P_{i}^{*}$ and $h_{i+1} \in H_{i+1}^{*}(i=1, \cdots, s)$. Let $P_{1}, \cdots, P_{s}$ be one-parameter subgroups of $G$ such that $\phi\left(P_{i}\right)=P_{i}^{*}(i=1, \cdots, s)$ and $P_{i} G_{n}=\{e\}$. Let $H_{i}=P_{i} \cdots P_{s} G_{n}(i=1, \cdots, s)$ and $H_{s+1}=G_{n}$. Since $\phi\left(P_{i} \cap H_{i+1}\right)$ $=\left\{e^{*}\right\}, P_{i} \cap H_{i+1}=P_{i} \cap G_{n}=\{e\}$ so that for all $h_{i} \in H_{i}, h_{i}=u_{i} h_{i+1}$ uniquely with $u_{i} \in P_{i}, h_{i+1} \in H_{i+1}(i=1, \cdots, s)$. Moreover, since $H_{i}=\phi^{-1}\left(H_{i}^{*}\right)(i=1, \cdots, s+1), H_{i+1}$ is a closed normal subgroup of $H_{i}(i=1, \cdots, s)$. It follows that topologically $H_{i}=P_{i} \times H_{i+1}$ so that $G=P_{1} \times P_{2} \times \cdots \times G_{n}$. Consequently $G_{n}$ is simply connected and, being abelian, is a Lie group. Repeated application of Lemma 4 yields the result that $G$ is a Lie group.

Proof of THE MaIn Theorem. Suppose now that $G$ is a connected, locally euclidean group with core $C$. Let $G^{*}$ denote the simply connected covering group of $G$, let $C^{*}$ denote the core of $G^{*}$, and let $\phi$ denote the natural homomorphism of $G^{*}$ onto $G$. It can be easily seen that $C^{*} C \phi^{-1}(C)$. By Lemma $1, G^{*} / C^{*}$ is simply connected. Hence by Lemma $5, G^{*} / C^{*}$ is a Lie group. But

$$
G / C=G^{*} / \phi^{-1}(C)=G^{*} / C^{*} / \phi^{-1}(C) / C^{*}
$$

so that $G / C$ is the image of the Lie group $G^{*} / C^{*}$ under a continuous homomorphism. It follows that $G / C$ is a Lie group.

Institute for Advanced Study and

SyraCUSE UNIVERSITY 